\title{
Optimum design and construction of a Zeeman slower for use with a magneto-optic trap
}

\author{
C. J. Dedman, J. Nes, T. M. Hanna, R. G. Dall, K. G. H. Baldwin, and A. G. Truscott
}

Citation: Review of Scientific Instruments 75, 5136 (2004); doi: 10.1063/1.1820524

View online: https://doi.org/10.1063/1.1820524

View Table of Contents: http://aip.scitation.org/toc/rsi/75/12

Published by the American Institute of Physics

\section{Articles you may be interested in}

A Zeeman slower design with permanent magnets in a Halbach configuration

Review of Scientific Instruments 82, 063115 (2011); 10.1063/1.3600897

A slow atom source using a collimated effusive oven and a single-layer variable pitch coil Zeeman slower

Review of Scientific Instruments 81, 013105 (2010); 10.1063/1.3276712

Designing and building a permanent magnet Zeeman slower for calcium atoms using a 3D printer

American Journal of Physics 83, 892 (2015); 10.1119/1.4930080

An introduction to Pound-Drever-Hall laser frequency stabilization

American Journal of Physics 69, 79 (2001); 10.1119/1.1286663

Investigation of different magnetic field configurations using an electrical, modular Zeeman slower

Review of Scientific Instruments 86, 103110 (2015); 10.1063/1.4934248

High flux source of cold rubidium atoms

Review of Scientific Instruments 76, 103101 (2005); 10.1063/1.2069651

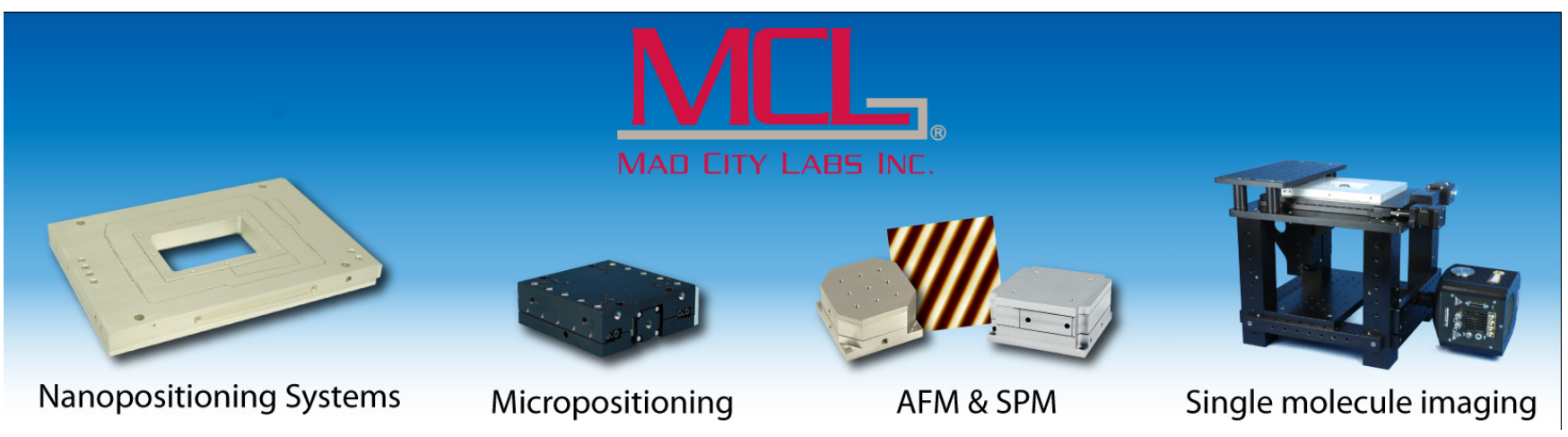




\title{
Optimum design and construction of a Zeeman slower for use with a magneto-optic trap
}

\author{
C. J. Dedman \\ Research School of Physical Sciences and Engineering, Australian National University, \\ Canberra ACT 0200, Australia \\ J. Nes \\ Research School of Physical Sciences and Engineering, Australian National University, \\ Canberra ACT 0200, Australia and Institut für Quantenoptik Universitat Hannover, Welfengarten 1, D-30167 \\ Hannover, Germany
}

\section{T. M. Hanna}

Research School of Physical Sciences and Engineering, Australian National University, Canberra ACT 0200, Australia and Clarendon Laboratory, Oxford University, Parks Road, OX13PV Oxford, United Kingdom

R. G. Dall, K. G. H. Baldwin, and A. G. Truscott ${ }^{\mathrm{a}}$

ARC Centre of Excellence for Quantum-Atom Optics, and Research School of Physical Sciences and Engineering, Australian National University, Canberra ACT 0200, Australia

(Received 2 May 2004; accepted 26 September 2004; published 10 November 2004)

A method for optimizing the design and construction of a Zeeman slower coil is presented. Unlike traditional designs, the measured magnetic field profile very accurately matches the desired field profile, enabling significant advantages for loading a magneto-optic trap. (C) 2004 American Institute of Physics. [DOI: 10.1063/1.1820524]

\section{INTRODUCTION}

In principle the idea of slowing an atomic beam is simple. A laser beam is directed against the motion of the atoms, the atoms absorb photons from the laser beam, and due to the photon recoil they experience, the atoms slow down. Unfortunately, as the atom's velocity decreases the Doppler shift out of resonance with the laser beam and this simple idea breaks down. A solution to this problem was first demonstrated by Phillips and Metcalf, ${ }^{1}$ and is known as a Zeeman slower. In Zeeman slowing, a spatially varying magnetic field produces a varying Zeeman shift which compensates the changing Doppler shift of the atoms, thus keeping them in resonance with the slower laser.

There are two main types of Zeeman slowers, one uses $\sigma^{+}$(Ref. 1) laser light the other $\sigma^{-}{ }^{2}$ The Zeeman slower we describe here is of the latter variety, however the design and construction methods are applicable to both. For a $\sigma^{-}$slower the relevant B-field profile is given by: ${ }^{3}$

$$
B(z)=B_{b}-B_{t} \sqrt{1-z / z_{0}},
$$

where $B_{b}$ is a constant bias field, $B_{t}$ is the magnitude of the spatially varying component (in our case $B_{b}=B_{t}$ ), and $z_{0}$ is the distance over which slowing takes place. The slower length is set by the deceleration, $a$, the laser beam imparts on the atom given by

\footnotetext{
a) Author to whom correspondence should be addressed; electronic mail: andrew.truscott@anu.edu.au
}

$$
a=\frac{\hbar k \Gamma}{2 m} \frac{s_{0}}{1+s_{0}+4\left[\delta_{0}+k v-\mu^{\prime} B(z) / \hbar\right]^{2} / \Gamma^{2}},
$$

where $\Gamma$ is the linewidth of the atomic transition, $m$ is the atomic mass, $s_{0}=I / I_{s}$ is the on-resonance saturation parameter (where $I$ is the laser intensity and $I_{s}$ is the atomic saturation intensity), $\hbar$ is Planck's constant, $k$ is the laser wave vector, $\delta_{0}$ is the laser detuning, $v$ is the velocity of the atom and $\mu^{\prime}$ is the magnetic moment for the atomic transition. The maximum possible deceleration, achieved when the atom is in resonance with the laser beam, sets the minimum length of slower and is given by

$$
a=\frac{\hbar k \Gamma}{2 m} \frac{s_{0}}{1+s_{0}}
$$

which saturates at high intensities to

$$
a=\frac{\hbar k \Gamma}{2 m} .
$$

However, it is usual when designing Zeeman slowers ${ }^{4}$ to design the slower assuming only some fraction, $\eta$, of this deceleration will be achievable in the laboratory. This gives the design some head room for practical constraints, such as imperfections in the Zeeman field or limited slower laser power. It follows then from Newton's laws of motion and Eq. (4) that to slow an atom from an initial velocity $v_{i}$ to a final velocity $v_{f}$ will require a slowing distance of 


$$
z_{0}=m \frac{v_{f}^{2}-v_{i}^{2}}{\eta \hbar k \Gamma},
$$

where $\eta$ is known as the design parameter. To design a Zeeman slower one chooses an $\eta$, and the length of the slower is then set by the initial and final velocities. When choosing $\eta$, the designer has to bear in mind two criteria: (i) how much slower laser power is available and (ii) how well can the field profile given by Eq. (1) be replicated. The first criterion is easily quantified, and from Eq. (3) it follows that for a particular $\eta$ we require a laser intensity given by

$$
\frac{I}{I_{s}}=\frac{\eta}{1-\eta} .
$$

The second criteria is not as straightforward to quantify. However, if one considers that for a Zeeman slower to work optimally we require, $\delta_{0}+k v=\mu^{\prime} B(z) / \hbar$ at each point along the slower, a relationship between $\eta$ and the slope of the slower magnetic field can be derived:

$$
\eta=\frac{2 m \mu^{\prime}}{\hbar^{2} k^{3} \Gamma} \frac{d B}{d z}\left(\frac{\mu^{\prime} B}{\hbar}-\delta_{0}\right) .
$$

This equation relates the fraction of deceleration required, $\eta$, for a given $d B / d z$. For a Zeeman coil with large $d B / d z$ fluctuations, more intensity is required to compensate these fluctuations. However, the coil will not operate above $\eta=1$ as an infinite amount of intensity is required [Eq. (6)]. Qualitatively Eq. (7) says, for a Zeeman slower to function correctly, it is necessary that the slope, $d B / d z$, of the field does not exceed the slope of Eq. (1), when the length is set to the theoretical minimum, i.e., $\eta=1$. Because typical Zeeman coils only roughly approximate the optimum profile given by Eq. (1) they are made significantly longer (e.g., 2.5 times longer, i.e., $\eta=0.4,{ }^{5,6}$ ) than the theoretical minimum length, to ensure that $d B / d z$ is always less than the theoretical value.

In our experiment we use metastable helium atoms $\left(\mathrm{He}^{*}\right)$ from a liquid helium cooled atomic discharge source ${ }^{7}$ with a most probable velocity $\sim 700 \mathrm{~m} / \mathrm{s}$, and thus $\eta \sim 0.4$ corresponds to $\mathrm{a} \sim 1.5 \mathrm{~m}$ length Zeeman slower. Besides the obvious inconvenience of such a long slower, minimizing the slower length also reduces the atomic beam divergence arising from the initial transverse velocity of the atoms. There is a second source of the beam divergence arising from diffusive heating due to the absorption of photons from the slowing laser which is independent of slower length and can be countered somewhat by focusing the slower laser towards the atomic beam source, as was performed in the present experiments. By minimizing the departure from the optimum profile, not only is the length minimized but the laser power required is also reduced. For both these reasons, it is necessary that the magnetic field profile follow expression (1) as closely as possible.

Similarly, the magneto-optic trap (MOT) ${ }^{8}$ should be located as close as possible to the end of the Zeeman slower. This is particularly important as the beam divergence increases very rapidly after the slower, due to the decreased axial speed of the beam. This presents a second challenge because, with a conventional Zeeman coil, the field extends considerably beyond the end of the slower coil. Because

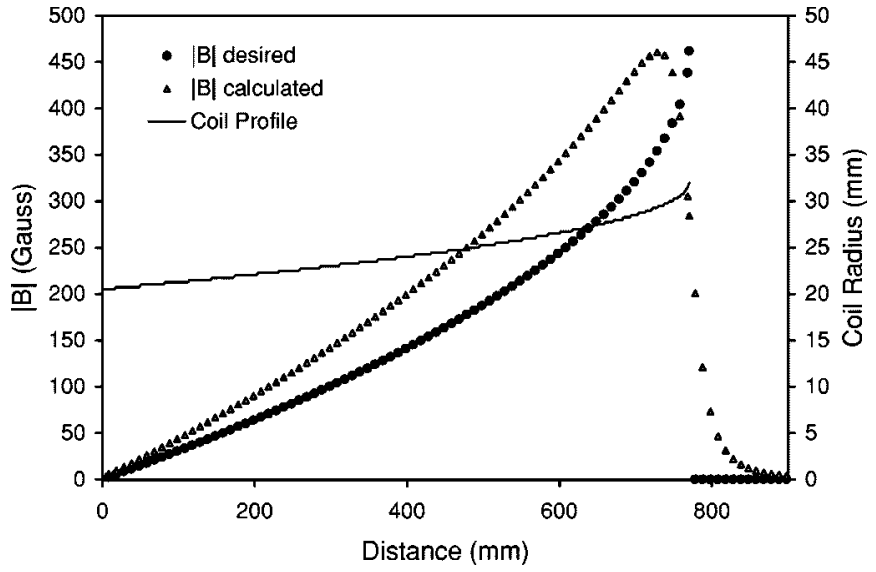

FIG. 1. Relevant Zeeman slower profiles. The desired B-field profile is shown, (-), as well as the calculated B-field when the number of winding layers, as a function of axial position, is simply made proportional to the desired field strength $(\triangle)$. Finally, the actual Zeeman coil profile that produces the $(\triangle)$ profile, is shown (solid line).

even a small stray field will impair trap operation, it is vital that the slower field falls to zero extremely rapidly beyond the end of the slower. Mu metal shielding has been used for this purpose, ${ }^{9}$ but this has the disadvantage of distorting the slower field, and greatly complicating the coil design. We have obtained excellent results with an "antiphase coil," described later in detail.

To our knowledge, there is no published information describing the optimum shape of coil required to accurately produce the required Zeeman magnetic field profile. The magnetic field strength within a long solenoid is proportional to the number of turns per unit length, $(N / l)$. It is apparently on this basis that Zeeman coils are commonly designed such that $(N / l)$ follows expression (1). That is, the number of winding layers, as a function of axial position, is simply made proportional to the desired field strength. Unfortunately, this does not give the required field, because the expression for the field within a long solenoid is not valid near the ends of the coil, and the expression is valid only for a uniform solenoid. Figure 1 shows the field that is obtained when the winding profile is made as described, and is compared with a plot of the desired field profile from expression (1). Evidently, the proportional winding field is a poor match to the desired field, so it is normally necessary to make the slower considerably longer than the theoretical minimum. Note that, expression (1) is valid, in principle, for slowing atoms down to zero velocity, but here we wish to slow atoms to $\sim 70 \mathrm{~m} / \mathrm{s}$, well within the capture range of our MOT. In such a case, the magnetic field profile should follow Eq. (1) almost to the peak, and then fall to zero as fast as possible.

In this article we describe the design and construction of a Zeeman slower with a design parameter $\eta=0.7$. Such a high design parameter allows a minimum amount of laser power to be used to achieve efficient slowing. In turn, a low laser power allows the Zeeman slowing beam to pass directly through the trapping region without perturbing trapped atoms, making the slower ideal for loading a MOT. Further- 


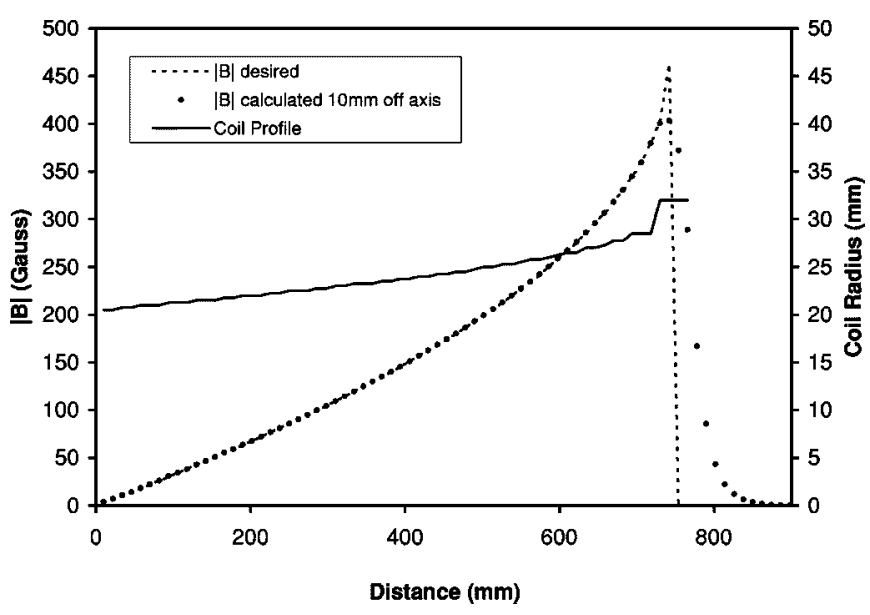

FIG. 2. Relevant Zeeman slower profiles $10 \mathrm{~mm}$ off axis. The desired B-field profile is shown, $(\cdots)$, as well as the calculated B-field when the Zeeman coil is wound optimally (-). Finally, the Zeeman coil profile that produces the $(\bullet)$ profile, is shown (solid line). Note that the program attempts to follow the desired profile of expression (1) only up to $400 \mathrm{G}$, after which the field should fall to zero as fast as possible.

more, we show from theoretical considerations that the slower is capable of application in a system requiring $\eta$ $=0.85$.

\section{CALCULATION OF OPTIMUM COIL PROFILE}

For any given shape of coil, it is relatively easy, with the aid of a computer, to use the Biot-Savart law to calculate the resultant magnetic field at any point in space. The reverse problem is more difficult. Given a required magnetic field profile, we wish to find the optimum coil shape to produce this field. A computer program was written to find the optimum coil shape. Before running the program, the overall coil length, coil inner diameter, wire diameter, and maximum number of winding layers areall assigned suitable fixed values. The task of the program is to find the optimum number of turns at each $z$ value, where the spacing between $z$ values is equal to the wire diameter. The Biot-Savart law is used to calculate the field strength at every $z$ value, by summing the field contribution from every turn, and the quality of the result is measured by the rms deviation between the desired and calculated fields.

The basic algorithm is as follows. At every $z$ value, the program calculates the effect of first adding one turn, and then subtracting one turn. If the rms deviation is improved, then the number of turns at that $z$ value is incremented or decremented accordingly. This is done repeatedly, with the result that the coil profile is slowly "built up," and converges to an optimum shape. In practice, it was found necessary to ensure that the coil profile remains monotonic-if this was not done then sensible results could not be obtained. Our requirement was to accurately produce the field profile given by Eq. (1), but the method may be useful in other applications, wherever there is a need to accurately produce a given magnetic field profile.

Figure 2 shows the optimum coil profile, as well as the desired field calculated from Eq. (1), and the calculated field resulting from the optimized coil. The results are remarkable

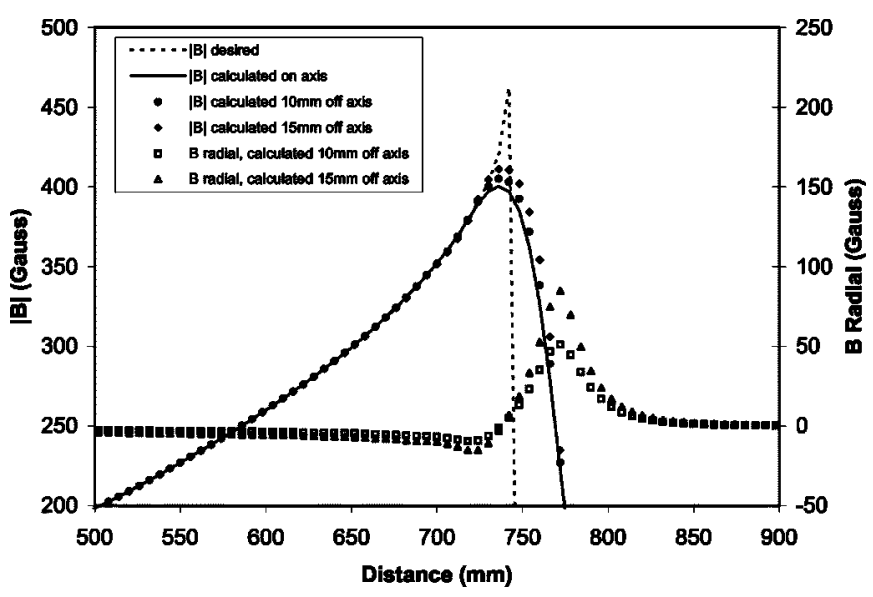

FIG. 3. Relevant Zeeman slower B-field profiles. The desired B-field profile is shown, $(\cdots)$, as well as the calculated $|B|$-field profiles on and off axis, and the corresponding radial B-field profiles.

for two reasons. First, the coil profile bears little resemblance to the field profile. Second, and even more surprising, is that the optimum coil profile is highly discontinuous in nature, with a high "cliff" and flat "plateau" in the region where the field strength rises smoothly to its maximum value. We cannot state definitively that the coil profile generated by the program is in fact the best possible profile, but it is certainly true that this coil profile gives excellent agreement between the calculated and desired fields, as illustrated in Fig. 2. Note that the program attempts to follow the desired profile of expression (1) only up to $400 \mathrm{G}$, after which the field should fall to zero as fast as possible. The rms deviation between calculated and desired fields is $\sim 0.07 \%$, such that the accuracy of the field produced by the Zeeman coil will in practice be limited by the mechanical precision with which we can construct the coil.

The on- and off-axis fields are slightly different. As the majority of the particles will be off-axis, the coil optimization calculations were performed $10 \mathrm{~mm}$ off-axis, see Fig. 2. The off-axis field has a significant radial component which is directly related to the rate-of-change of field strength, $d B / d z$, and is therefore quite significant at the end of the slower, where the field is changing rapidly. Figure 3 shows the small variation in $|B|$ as a function of radial offset, as well as the significant radial component near the end of the slower. The Zeeman shift is a function only of overall field strength, rather than the axial or radial field components, therefore, the program optimizes coil shape such that $|B|$, calculated $10 \mathrm{~mm}$ off axis, is matched to the desired $\mathrm{B}$ profile of expression (1). Performing the required off-axis calculations involving both axial and radial field components is numerically intensive, requiringextensive use of lookup tables to achieve satisfactory execution time on a $650 \mathrm{MHz}$ PC.

As briefly mentioned, an "antiphase coil" (A/C) was constructed to cancel out the stray field at the exit of the Zeeman coil. Of course, any $\mathrm{A} / \mathrm{C}$, or even conventional Helmholtz coils, can produce perfect cancellation at a single point, but this is not particularly useful, as our requirement is to minimize the stray field over a significant distance, in the vicinity of the MOT. Stating this in a different way, near the trap center, both $|B|$ and $d B / d z$ must have small values. This 


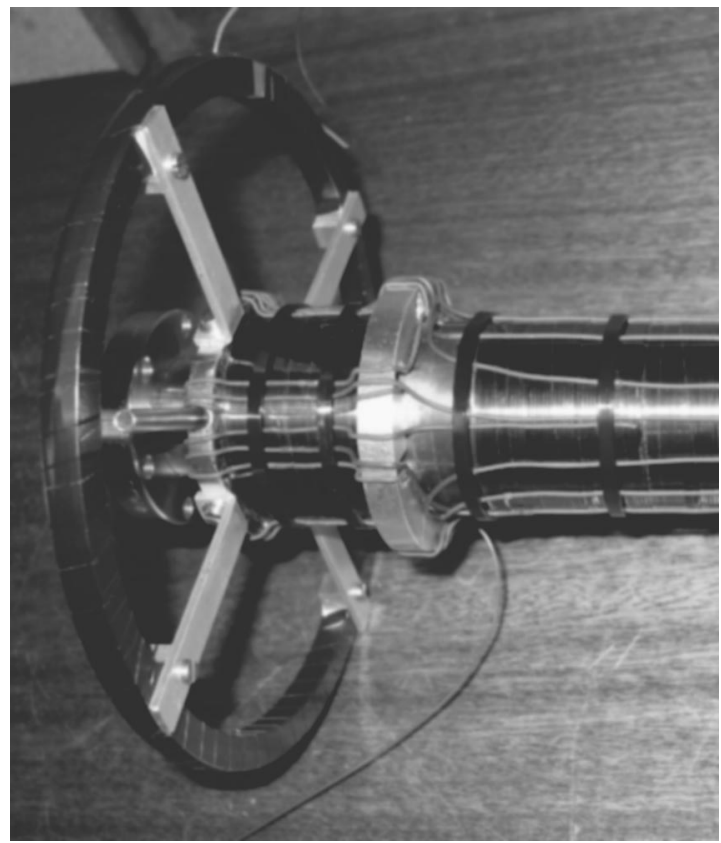

FIG. 4. Picture of the "antiphase-coil" located at the end of the Zeeman slower.

is achieved by optimizing the diameter, axial position, and strength of the $\mathrm{A} / \mathrm{C}$, and this optimization is performed with the same program that designs the Zeeman coil. The A/C significantly perturbs the Zeeman field, so the design algorithm is as follows. First, the Zeeman coil is optimized without the $(\mathrm{A} / \mathrm{C})$, and then the $\mathrm{A} / \mathrm{C}$ coil is optimized to minimize the stray field in the trap region. Then the Zeeman coil is optimized in the presence of the $\mathrm{A} / \mathrm{C}$ field, the $\mathrm{A} / \mathrm{C}$ coil is reoptimized, etc. It was found that the optimum $\mathrm{A} / \mathrm{C}$ for our Zeeman slower is $210 \mathrm{~mm}$ in diameter, located axially very close to the end of the Zeeman coil and with a strength of 220 A-turns. The A/C is clearly visible in the image of Fig. 4. The antiphase coil is connected in series with the Zeeman coil, so that the field cancellation is correctly maintained when the Zeeman coil current is altered. The precise number of turns was empirically "tuned" to obtain the (very small) calculated stray field.

\section{ZEEMAN SLOWER MAGNETIC FIELD MEASUREMENTS}

The on-axis magnetic field within the Zeeman coil was measured using a Bell model 640 Hall-probe gauss meter. It would have been difficult to measure the off-axis field, as this would require the measurement of both axial and radial field components. Figure 5 shows the excellent agreement between the measured and calculated on-axis fields. Given the excellent agreement of the on-axis data, we are confident that the off-axis field is also in good agreement with calculations. The stray field outside of the coil was measured with a Meda model FVM-400 digital magnetometer. Again, the agreement is good, and the strength of the stray field in the vicinity of the MOT is negligible at an average value of around $25 \mathrm{mG}$. In the absence of the antiphase coil, the stray field at the trap center is around $1900 \mathrm{mG}$.

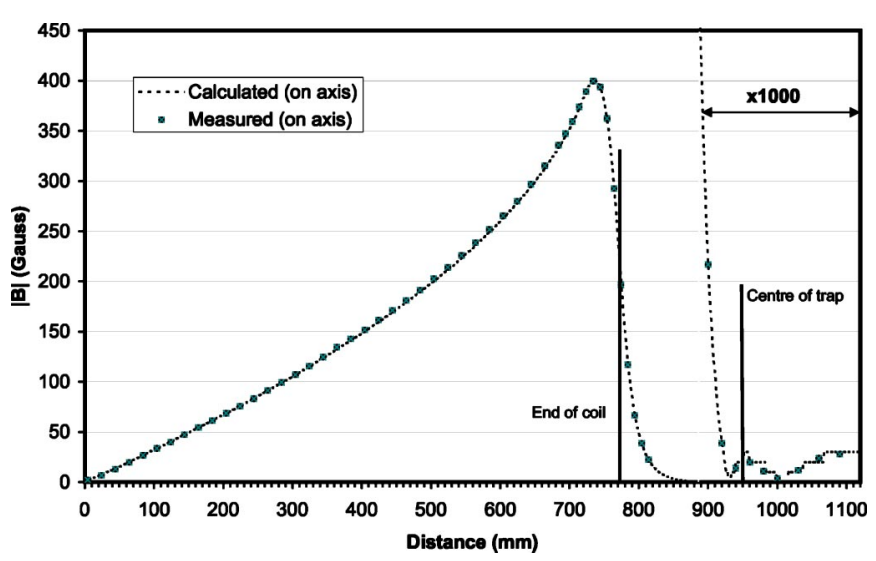

FIG. 5. Measured and calculated on-axis Zeeman slower profiles. The B-field in the magneto-optic trap (MOT) region has been scaled up by a factor of a thousand for clarity.

\section{CONSTRUCTION DETAILS}

The method of winding our Zeeman coil is unusual. Normally, multilayer coils are constructed by winding left-toright, then returning from right to left, etc. At first it might be thought that each turn from a particular winding layer would drop exactly into the "groove" formed by adjacent turns of the previous layer, and this does happen to some extent. However, because the turns on adjacent layers "slant" in opposite directions, the turns on each layer must "cross over" the turns from the previous layer, and cannot drop into the "groove" for an entire turn. The practical result is that, after ten layers, the winding starts to become lumpy and ragged, thus compromising the precision of the magnetic field profile. To avoid this problem, all layers are wound in the same direction, from left to right, with the result that the last layer is as perfectly uniform as the first. The thermal conductivity of the coil is also improved, because the turns are packed closer together. The disadvantage of this scheme is that the right-hand end of each layer has to be externally connected back to the left-hand end of the next layer, after the coil winding is completed. These external connecting wires are clearly visible in Fig. 4. For large coils, square section wire could be employed with similiar advantages. However, square section wire is not available down to $0.5 \mathrm{~mm}$, as required here.

Another innovation, in this design, is the use of short, double-pitch windings to halve the number of required layers, for a given "ripple" in the magnetic field. The program actually calculates the details for a coil having 46 layers, which reduces the ripple to a negligible value. However, winding 46 layers would be very tedious, so instead the main coil is physically wound with only 23 layers. Short, doublepitch "trim-windings" are then added at the end of each layer, giving identical performance to a 46 layer coil. Details are visible in the image of Fig. 6. The trim windings are electrically in series with the main winding. The coil former is water cooled, and the windings remain close to room temperature at the normal operating current of $1.05 \mathrm{~A}$. The average coil diameter was made as small as possible to minimize the stray field outside of the coil. The coil inner diameter is $41.5 \mathrm{~mm}$, and the coil outer diameter is mini- 


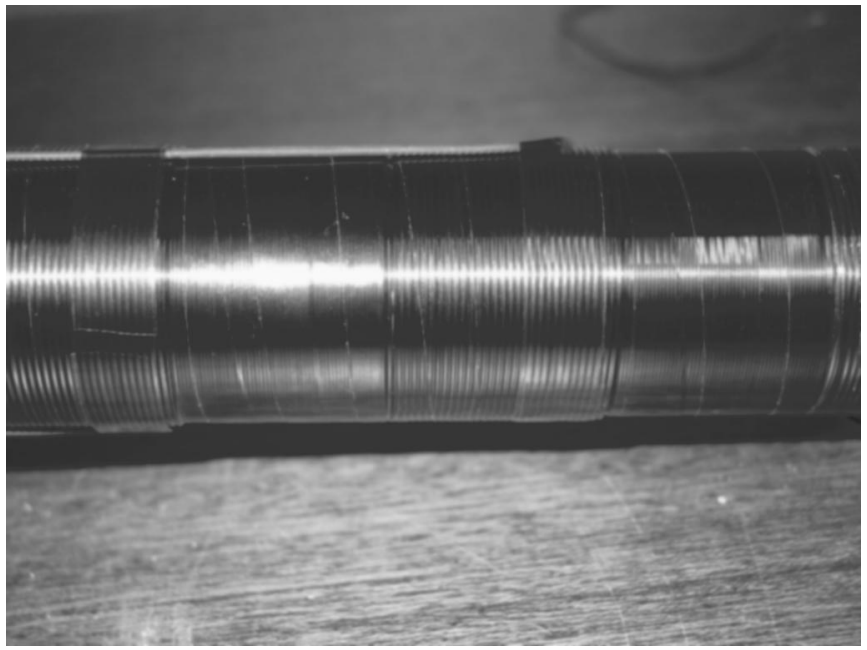

FIG. 6. Photograph of the Zeeman slower, showing double-pitch trim windings.

mized by using the finest gauge of wire $(0.5 \mathrm{~mm}$ diameter.) consistent with acceptable resistive heating of the coil.

\section{MODELING OF THE ZEEMAN SLOWER}

To model the performance of our Zeeman slower we simulate the trajectories of atoms passing through the slower. The simulation is two-dimensional, longitudinal, and radial, and interpolates the B-field that an atom experiences from a lookup table produced from an experimentally determined B-field profile. The model numerically integrates Eq. (2) using a Runge-Kutta method, thereby modeling only the conservative part of the scattering process. Spontaneous emission is added in a random manner at the end of each integration period.

Initially, atoms are assumed to have a random initial transverse velocity in the range $\pm 3 \mathrm{~m} / \mathrm{s}$, and a longitudinal velocity in the range of $700-500 \mathrm{~m} / \mathrm{s}$, simulating the experimentally determined conditions of atoms arriving from our collimated source. The simulation also includes noise sources, such as current noise $(\sim 0.25 \%)$ on our Zeeman slower power supply and laser detuning jitter $( \pm 300 \mathrm{kHz})$. Finally, to properly model the convergence of the laser beam used, we use a position dependent transverse scattering force.

\section{RESULTS AND DISCUSSION}

To test the performance of our slower we performed a time-of-flight (TOF) experiment. The counterpropagating slowing laser beam was reflected into the Zeeman slower using a mirror with a $1 \mathrm{~mm}$ diameter central hole through which the slowed atoms passed, following the method of Ref. 5. Downstream of the mirror the TOF equipment was mounted, comprising a rotating disk with a slot (the "chopper wheel") to gate the atoms, and an optical detection system to provide triggering for the data-acquisition software. The atoms travel $610 \mathrm{~mm}$ from the chopper wheel to a channeltron, where the $\mathrm{He}^{*}$ atoms, due to their internal energy of $\sim 20 \mathrm{eV}$, can be counted with almost unit efficiency.

TOF spectra with and without Zeeman slowing are shown in Fig. 7. We are able to remove $71 \%$ of atoms below

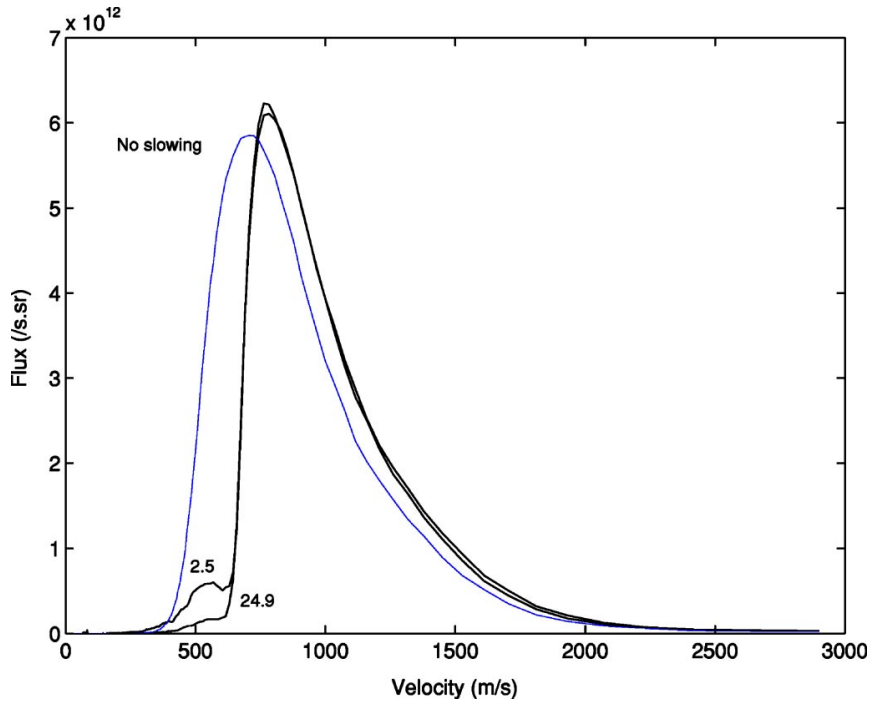

FIG. 7. Demonstration of Zeeman slowing for 2.5 and $24.9 \mathrm{~mW}$. A TOF spectrum with no slowing is shown for comparison.

$700 \mathrm{~ms}^{-1}$ with $2.5 \mathrm{~mW}$ of slowing light, and $82 \%$ with $24.9 \mathrm{~mW}$, corresponding to $32 \%$ and $38 \%$ of the total flux, respectively. The capture velocity can be seen to be $\sim 700 \mathrm{~ms}^{-1}$, a result of power broadening around the resonant velocity of $690 \mathrm{~ms}^{-1}$.

Figure 8 shows the detailed slowed atom TOF distribution observed for $2.5 \mathrm{~mW}$ laser power (-) and the corresponding numerical simulation of the system (solid line). With the slower laser beam on, a slow atom peak is seen at around $80 \mathrm{~m} / \mathrm{s}$ in good agreement with the theoretical predictions.

To characterise our Zeeman slower we have performed measurements of its effect on $\mathrm{He}^{*}$ beams for a range of slowing powers $\left(0.96-24.9 \mathrm{~mW}\right.$, corresponding to $s_{0}$ $=10-264$ ) and for a variety of magnetic fields (peak field range $377.7-432.2 \mathrm{G}$, corresponding to a range of $\eta$ of $0.68-0.70)$.

Figure 9 shows the results of varying the power in the

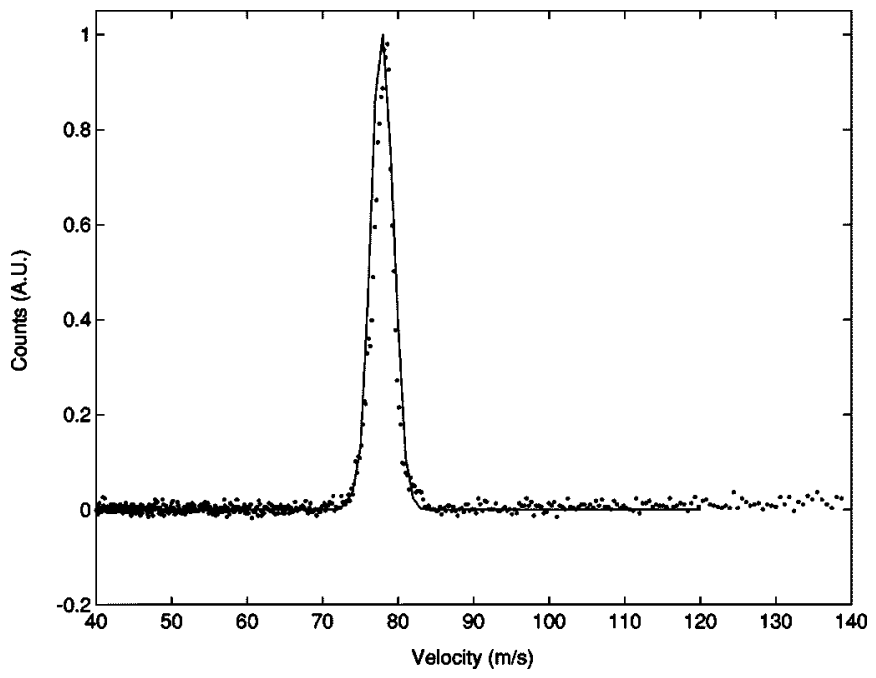

FIG. 8. Experimentally measured slow atom peak using our Zeeman slower (O) as well as the result from our numerical simulation of the slower (solid line). 


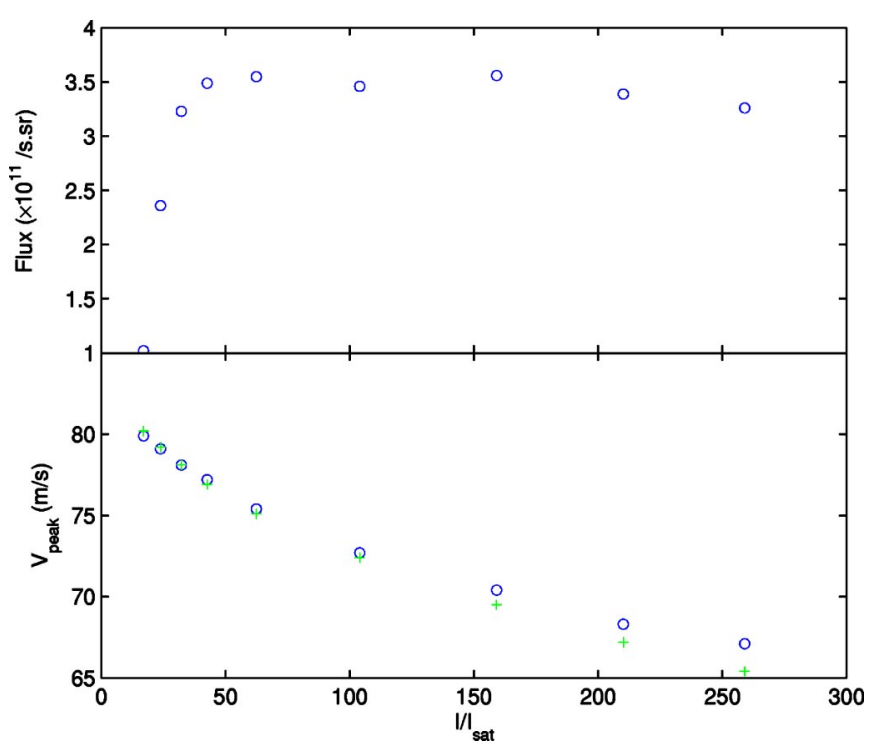

FIG. 9. Variation in the properties of the slowed beam with slowing laser power: (a) total flux, and (b) peak velocity of the slowed atoms measured experimentally $(\bigcirc)$ and determined theoretically $(+)$.

slowing laser beam, keeping all other parameters constant. Figure 9(a) shows that the flux in the slower beam is approximately constant for powers above $2.5 \mathrm{~mW}$, corresponding to $s_{0}=26$. Power broadening allows us to significantly reduce the peak velocity (to $68 \mathrm{~ms}^{-1}$ in the case of the highest power we tested), shown in Fig. 9(b). Also shown in Fig. 9(b) are corresponding simulations of the system. Since the exact on-axis laser power is not known, due to the hole in the reflecting mirror, a scaling factor is used in our simulations. We find that simulations of the system with a factor of four less power give good agreement with our experimental results. This indicates that we should be able to use $<1 \mathrm{~mW}$ to slow atoms to within the capture range of our MOT. This compares favorably with a similar metastable helium experiment ${ }^{10}$ that used $20 \mathrm{~mW}$ in a beam of similar $1 / e^{2}$ radius.

The results of varying the magnetic field to alter the final velocity are shown in Fig. 10. The magnetic field was varied for two powers of slowing light: 2.5 and $26 \mathrm{~mW}$. Figure 10(a) shows the flux observed, the apparent decline being due to increased divergence of the slower beam as the peak magnetic field is increased. Flux multiplied by $v_{0}^{2} / v^{2}$ (where $v_{0}$ is the peak velocity of the flux measured at lowest magnetic field, and $v$ is the peak velocity of the flux at the relevant magnetic field) is shown in Fig. 10(b). This correction accounts for the greater divergence and thus lower flux of slower atom beams. There is an evident decline in scaled flux for $26 \mathrm{~mW}$ of slowing light, but that for $2.5 \mathrm{~mW}$ shows little difference in the performance of the Zeeman slower within the range of conditions tested. The decrease in flux, seen at higher power, is probably a result of increased transverse heating. Figure 10(c) shows the measured peak velocities for both powers as well as corresponding simulations of the system. Once again our simulation of the Zeeman slower is in good agreement with the experimental results.

Our slower is $\sim 750 \mathrm{~mm}$ long and was designed to slow atoms with velocities up to $700 \mathrm{~m} / \mathrm{s}$, this equates to a slower

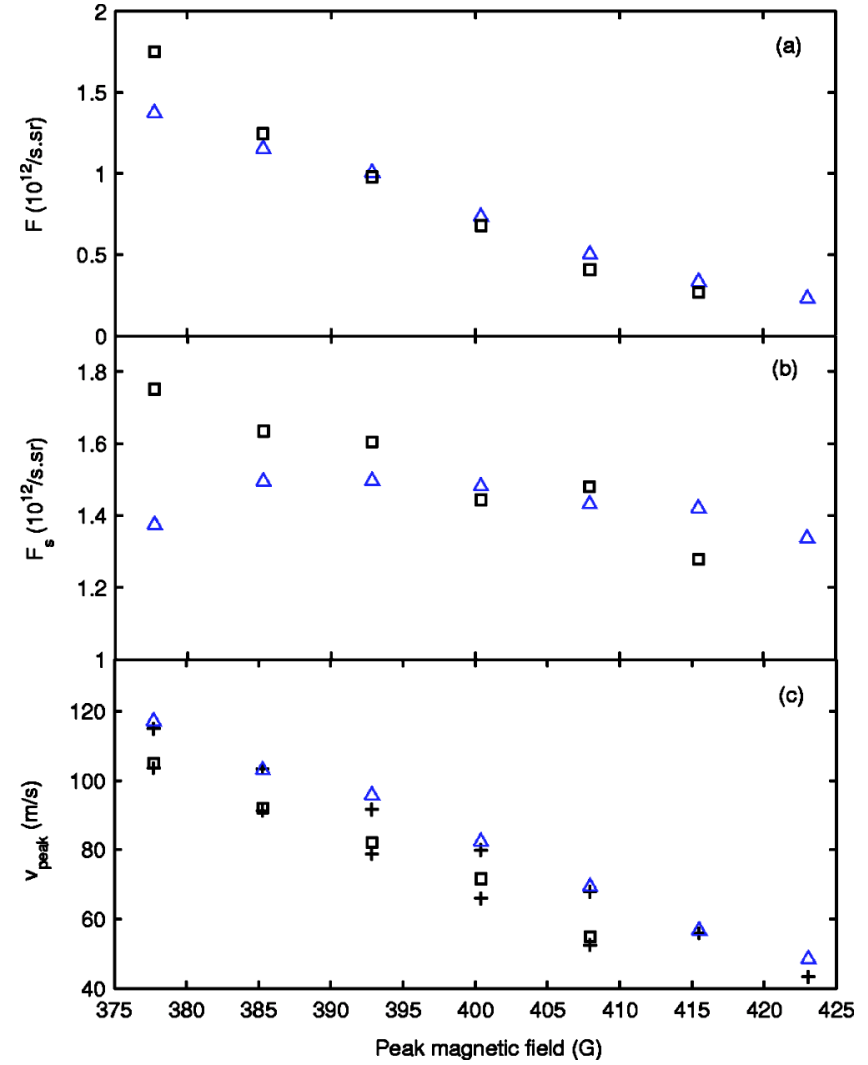

FIG. 10. Variation in slowed beam properties with magnetic field for $2.5 \mathrm{~mW}(\triangle)$ and $26 \mathrm{~mW}(\square)$ of slowing light: (a) total flux, (b) flux scaled according to velocity, and (c) peak velocity of the slowed atoms measured experimentally, and determined theoretically $(+)$.

efficiency $\sim \eta=0.7$. However, by changing the laser detuning, $\delta_{0}$, we can slow higher velocity atoms and in turn change the effective $\eta$ of the slower. We are of course limited by the two criteria mentioned previously, laser intensity and $d B / d z$. For the $2^{3} S_{1}-2^{3} P_{2}$ transition of $\mathrm{He}^{*} I_{s}$ $=0.17 \mathrm{~mW} / \mathrm{cm}^{2}$, so even for $\eta=0.95$ only a relatively low intensity of $\sim 3.2 \mathrm{~mW} / \mathrm{cm}^{2}$ is required, which is not a limi-

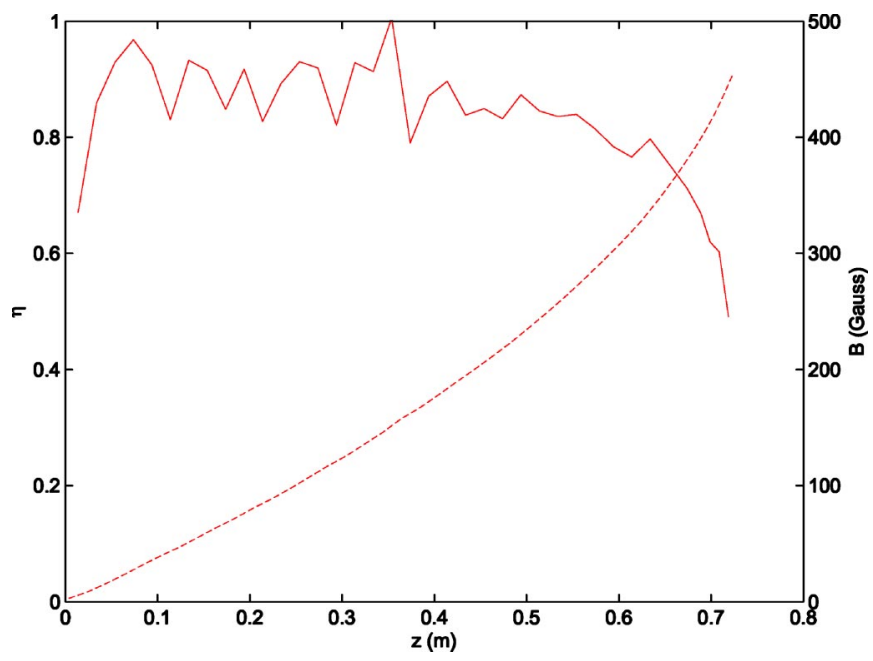

FIG. 11. Plot of $\eta$ as a function of axial distance $z$ for our Zeeman slower. Also plotted is the on-axis experimentally measured B-profile, shown as a dashed line. 
tation to our system. On the other hand a poorly wrapped slower with sharp changes in B field would limit the performance of our system.

Figure 11 shows $\eta$ as a function of $z$ for our slower, given by Eq. (7), for $\delta_{0}=k v_{i}=k \times 760 \mathrm{~m} / \mathrm{s}$, where we have used the on-axis experimentally measured B profile, shown as a dashed line. It is clear for these parameters that $\eta<1$ at all points along the slower, and thus the slower should function correctly. The effective $\eta$ for this system, as determined by Eq. (5) is 0.85 . We have qualitatively confirmed that our slower works for an $\eta=0.85$ by efficiently loading a MOT with the relevant settings (i.e., $\delta_{0}=k \times 760 \mathrm{~m} / \mathrm{s}$ and $|B|_{\max }$ $=455 \mathrm{G}$.

The independence of slowed flux upon laser power within the range tested allows an estimate of the total flux into the MOT to be made based simply on the measured flux of atoms, an assumed capture area for the MOT of $2 \mathrm{~cm}$ diameter, and a separation between the trap and slower of
$150 \mathrm{~mm}$. This gives an estimate of $\sim 10^{10}$ atoms/s being loaded into the MOT, a rate which has subsequently been verified experimentally.

${ }^{1}$ W. D. Phillips and H. Metcalf, Phys. Rev. Lett. 48, 596 (1982).

${ }^{2}$ T. E. Barrett, S. W. Dapore-Schwartz, M. D. Ray, and G. P. Lafyatis, Phys. Rev. Lett. 67, 3483 (1991).

${ }^{3}$ S. K. Mayer, N. S. Minarik, M. H. Shroyer, and D. H. McIntyre, Opt. Commun. 210, 259 (2002).

${ }^{4}$ H. J. Metcalf and P. Van der Straten, Laser Cooling and Trapping (Springer, New York, 1999).

${ }^{5}$ W. Lu, Ph.D thesis, Australian National University, 1999.

${ }^{6}$ I. Courtillot, A. Quessada, R. P. Kovacich, J.-J. Zondy, A. Landragin, A. Clairon, and P. Lemonde, Opt. Lett. 28, 468 (2003).

${ }^{7}$ J. A. Swansson, K. G. H. Baldwin, M. D. Hoogerland, A. G. Truscott, and S. J. Buckman, Appl. Phys. B: Lasers Opt. 79, 485 (2004).

${ }^{8}$ E. L. Raab, M. Prentiss, A. Cable, S. Chu, and D. E. Pritchard, Phys. Rev. Lett. 59, 2631 (1987).

${ }^{9}$ K. E. Strecker, Master's thesis, Rice University, 2002.

${ }^{10}$ W. Rooijakkers, W. Hogervorst, and W. Vassen, Opt. Commun. 135, 149 (1997). 